\title{
Searching for trouble?
}

The search for signs of intelligent life elsewhere in the Universe has been underway since the pioneering efforts of Frank Drake in the 1960s. Most prominently, the privately funded SETI Institute has scrutinized radio signals for three decades and found nothing, despite widespread belief that the Universe must be teeming with other civilizations. Fermi's paradox - the conspicuous lack of any signs of other intelligences, despite belief that they must exist - lives on.

Of course, the SETI search has only looked at about one in 50 million stars in the Milky Way, and may have simply looked at the wrong ones, or at signals in the wrong frequencies. The programme has also limited itself to a passive search, scanning the sky with increasingly sensitive devices and processing the resulting volumes of data. Active efforts to make contact with extraterrestrials by signal transmission have been far less common. Drake himself sent out a weak message in 1974, and later (reportedly) regretted the act. A few other amateurs have sent out signals, but as yet humanity has not undertaken any serious programme of transmitting strong signals towards specific targets in the hope that someone, or something, might detect them and infer our presence.

Such an approach does seem inherently risky, given our complete lack of information about the kinds of civilizations that may be out there, and the attitude they may take towards us. Yet not everyone shares this view, and there's been a recent movement to initiate a technologically advanced effort to send out signals using, for example, the 305 metre radio telescope at Arecibo, Puerto Rico. The scientists behind the movement - they call it METI, for Messaging to Extra-Terrestrial Intelligence - argue that this is the logical next step now that a passive search has failed to find anything for so long.

The idea is controversial, and rightly so. With access to the right transmitting devices, even one person could unilaterally take a momentous step affecting the future of all humanity, without any broad global discussion or agreement. At worst, the consequences could be catastrophic, as any civilization detecting our presence is likely to be technologically very advanced, and may not be disposed to treat us nicely. At the very least, the idea seems morally questionable. In a

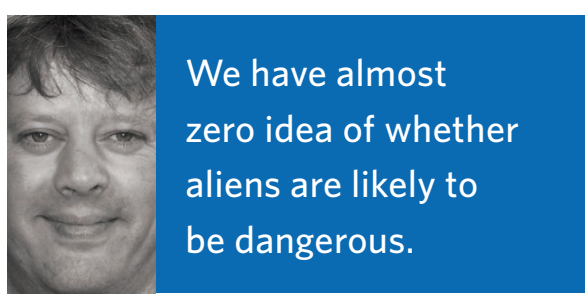

recent paper published on the arXiv (https://arxiv.org/abs/1605.05663), John Gertz of the Foundation for Investing in Research on SETI Science and Technology has tried to raise awareness of the issue - and to make the case for continuing with the more conservative passive approach.

You might think that active signalling isn't really risky because the radio waves we use for communications have already been leaving Earth and travelling out into space since the 1930s. As of now, as Gertz points out, such signals have swept over the nearest 7,000 stars. However, this leakage is quite weak; a telescope of Arecibo's sensitivity on a planet in any of those 7,000 star systems would not be able to detect it, although a more sensitive one, of course, might. If aliens' telescopes are like ours, then the chance of our leakage being detected could well dwindle over time as ever more of our communications take place through cables, or over directed satellite links, rather than through simple atmospheric transmission.

Hence, we may be at the end of a window of time during which our normal communications technology has emitted a significant detectable signal. Not so if we begin broadcasting a signal from Arecibo, as proposed by METI supporters Douglas Vakoch and Seth Shostak. Such a signal would be some 100,000 times stronger than the leakage signal of previous decades. It's true, as some METI advocates argue, that Arecibo in its operation as an asteroid-tracking radar has already sent out such signals. Yet these tracking signals wander over the sky, making them very much unlike a beam that would be focused over significant periods of time on a single star system.

It's hard not to see this as gratuitously risky, especially as it would be undertaken for no other reason than to satisfy our deep intellectual curiosity. We have almost zero idea of whether aliens are likely to be dangerous, although the single history of evolving biological life that we know of - here on Earth - carries a strong theme of violent conflict, perpetual battle for resources and the oppression of weaker groups by stronger ones. Yet, as Gertz points out, many METI enthusiasts follow a naive faith that any advanced alien civilization will also be morally advanced and will aim to interact with us peacefully.

In fact, one idea favoured by METI-ists is a version of the so-called zoo hypothesis put forward as one possible solution of the Fermi paradox. This idea holds that we've never seen aliens, despite their presence throughout the Universe, because the aliens have decided to leave us alone. As a result of their moral advancement, alien civilizations view lesser-developed parts of the Universe - those with still young civilizations - as wilderness areas or nature preserves to be protected and left undisturbed, at least until the intelligences within them reach out with signals showing their desire to make contact. Having made this assumption, METI-ists then assert that it's time for us to do so.

Gertz is right that this is nothing more than a grand and very dangerous assumption with absolutely no backing. And this is among the reasons that many scientists have strongly criticized the METI proposals, which have, so far, been roundly rejected. It seems far wiser to wait, and continue the developing passive search programme already established by the SETI Institute, which is due to accelerate in the near future. Just last year, businessman Yuri Milner donated US\$100 million over ten years to help boost the project. One day it may even get public funding.

We have no understanding of how close passive SETI search may be to discovery. It could come with the next advance in sensitivity, as happened with the search for gravitational waves. Meanwhile, technology is driving rapid progress in computing resources, in the knowledge of the locations of exoplanets that are likely to be suited to life and in astronomical technique in general.

Patience must be among the qualities any mature and advanced civilization should possess. We would do well to exercise such patience, while ensuring that the least patient among us cannot access the technology that would let them take matters into their own hands.

MARK BUCHANAN 